\title{
I've got 99 problems but a phone ain't one: Electronic and mobile health in low and middle income countries
}

\author{
Pratap Kumar, ${ }^{1,2}$ Chris Paton, ${ }^{3}$ Doris Kirigia ${ }^{4}$
}

\begin{abstract}
${ }^{1}$ Institute of Healthcare
Management, Strathmore

Business School, Nairobi,

Kenya

${ }^{2}$ Health-E-Net Limited, Nairobi, Kenya

${ }^{3}$ Centre for Tropical Medicine and Global Health, Nuffield

Department of Medicine, University of Oxford, Oxford, UK

${ }^{4}$ KEMRI-Wellcome Trust Research Programme, Nairobi, Kenya
\end{abstract}

Correspondence to Dr Pratap Kumar, Health-E-Net Limited, P.O. Box 357-00606, Nairobi, Kenya;

pratap@health-e-net.org

Received 7 September 2015

Revised 4 May 2016

Accepted 16 May 2016

Published Online First

13 June 2016

CrossMark

To cite: Kumar $P$, Paton $C$, Kirigia D. Arch Dis Child

2016:101:974-979.

\begin{abstract}
Mobile technology is very prevalent in Kenya-mobile phone penetration is at $88 \%$ and mobile data subscriptions form $99 \%$ of all internet subscriptions. While there is great potential for such ubiquitous technology to revolutionise access and quality of healthcare in low-resource settings, there have been few successes at scale. Implementations of electronic health (e-Health) and mobile health (m-Health) technologies in countries like Kenya are yet to tackle human resource constraints or the political, ethical and financial considerations of such technologies. We outline recent innovations that could improve access and quality while considering the costs of healthcare. One is an attempt to create a scalable clinical decision support system by engaging a global network of specialist doctors and reversing some of the damaging effects of medical brain drain. The other efficiently extracts digital information from paper-based records using low-cost and locally produced tools such as rubber stamps to improve adherence to clinical practice guidelines. By bringing down the costs of remote consultations and clinical audit, respectively, these projects offer the potential for clinics in resource-limited settings to deliver high-quality care. This paper makes a case for continued and increased investment in social enterprises that bridge academia, public and private sectors to deliver sustainable and scalable e-Health and m-Health solutions.
\end{abstract}

\section{INTRODUCTION}

Electronic health (e-Health), a broad term inclusive of mobile health (m-Health) that encompasses electronic-supported processes and communication in healthcare, is making forays in areas as diverse as medical recordkeeping, clinical decision support and coordination of care in almost every country and setting. ${ }^{1-3}$ Coupled with mobile connectivity, e-Health has been trumpeted as a means of revolutionising access to healthcare. It is broadly expected that such technology will positively impact access and quality of healthcare services in low and middle income countries (LMICs). ${ }^{4}$

The mobile technology revolution is very real in Kenya. In a country of 44 million people, there are almost 38 million mobile phone subscriptions (penetration of over 88\%), about 29 million mobile money transfer subscriptions and an estimated 32 million internet users; mobile data subscriptions form $99 \%$ of all internet subscriptions. ${ }^{5}$ The rest of the continent is not far behind. However, this advance in connectivity has yet to reach its potential in healthcare. Technology initiatives are indeed making significant differences to both healthcare delivery and management in Kenya and other countries, ${ }^{4}$ These advances are, however, primarily at limited scale and mostly focused on well-funded, vertical disease programmes like HIV/AIDS. ${ }^{2-4}$

Why has e-Health not yet engendered the 'revolution' in access to and quality of medical care in LMICs that was expected? Some reasons include traditional challenges such as the severe shortfall of human resources that limit any initiative to increase access to care. ${ }^{4}$ Others include 'human' issues that limit the buy-in and use of new technologies by their stakeholders. ${ }^{6}$ Variously referred to as 'human-centred design' or 'process innovation', attention to existing workflows, incentive structures, participation of users at multiple levels of implementation and broader links into the health system are all important issues that can determine the success of e-Health interventions. Even if a pilot is considered 'successful', small-scale implementations often lack programmatic evidence to inform the scale-up of e-Health interventions. ${ }^{8}$ In this article, we detail some of the broader barriers to technology adoption in LMIC healthcare systems and possible ways to addressing them.

\section{ADOPTION OF E-HEALTH SYSTEMS IN HIGH-INCOME COUNTRIES VERSUS LOW-INCOME COUNTRIES}

Over the last two decades, e-Health systems such as Electronic Medical Records (EMRs), Picture Archiving and Communication Systems (PACS) and Patient Administration Systems (PAS) have been widely adopted in high-income countries. This adoption has been achieved at a high cost, and different drivers of adoption, from medical coding and billing to participating in government programmes, have resulted in systems that are often difficult to use at the point of care and limited in their ability to share clinical information across facilities. ${ }^{9}{ }^{10}$ Increasing emphasis is therefore being placed on issues of interoperability and clinical usability. ${ }^{11}$

To address the problems with sharing data and allowing e-Health systems to communicate with each other, a process of international standardisation has emerged. Led by institutions such as Health Level 7 (HL7), Integrating the Healthcare Enterprise (IHE), Digital Imaging and Communications in Medicine (DICOM) and the International Health Terminology and Standards Development Organisation (IHTSDO), these standards have achieved high levels of adoption. 
However, much work is still needed to ensure seamless integration of systems and secure sharing of health data. By using internationally agreed standards, the global marketplace for e-Health systems can grow with less friction and benefit from pooling expertise from different countries. The market in LMICs, where e-Health systems are just starting to be implemented at scale, could especially benefit from such efforts.

In countries such as Kenya, hospital-level systems are now beginning to be implemented following several years of successful implantation of clinic-level EMR systems. One of these systems, OpenMRS, has been installed in multiple East African countries and in over 300 clinics in Kenya. ${ }^{12}$ However, the majority of the documentation of the implementation of such systems has focused on functionality and organisational and technical infrastructure. As highlighted in a recent review of 47 articles that reported success criteria for EMR system implementation in low-resource settings, training criteria (sufficient training and skilled personnel) only constituted $10 \%$ of 381 success criteria collected; political, ethical or financial considerations were rarely reported. ${ }^{13}$ While it is likely that low-income countries will take a different approach to the large-scale adoption of e-Health systems that has been seen in high-income countries, there are similar concerns around issues such as interoperability, privacy and sustainability that must be addressed while generating an evidence base for scale-up.

\section{TELEMEDICINE AND TABLETS-CHALLENGES AND SOLUTIONS TO GAPS IN HUMAN RESOURCES FOR HEALTH}

There are large shortfalls of human resources for health (HRH) in LMICs. The WHO recommends a minimum health worker to population ratio of 23 per $10000 .{ }^{14}$ This ratio in the 47 counties of Kenya varies from a high of 15.6 to as low as 1.2 (figure 1). ${ }^{15}$ Similar shortfalls and disparities in HRH exist for secondary care services, as measured by proxy through the number of hospital beds and cots available to the population. The number of hospital beds (across public and private sectors) ranges from approximately 34.8 per 10000 people to as few as 3.9-an almost 10 -fold difference across Kenya's counties. ${ }^{16}$

LMICs also face a large and growing burden of noncommunicable diseases (NCDs) alongside a continued presence of infectious diseases. With the latter still representing the majority investment into healthcare, the limited ability to manage NCDs like hypertension, diabetes, heart disease and cancer is a looming challenge for LMIC health systems. ${ }^{17}$ For example, Kenya has just 12 oncologists and 64 orthopaedic surgeons for a population of almost 45 million (author's compilation). Most of the few specialists (83\% of the oncologists and $73 \%$ of the orthopaedic surgeons) practise in Nairobi, which is home to just $8 \%$ of the Kenyan population. Exacerbating this disparity, secondary care services are mainly delivered via the private sector, and most of the costs of these services borne out-of-pocket. ${ }^{18}$ When combined with large geographic scales, poor rural roads and high costs of transport, access to services beyond primary healthcare is mostly restricted to urban and/or affluent populations.

Telemedicine has been viewed as a potential means of overcoming geographic disparities in access to care. The 'promise' has been that telemedicine could be more practical, affordable and sustainable than traditional programmes and attract physicians, nurses and other HRH to work in remote areas (or on health issues in these areas) on a short-term or long-term basis. ${ }^{19}$ This is yet to be demonstrated at scale, especially in LMICs due to some significant limitations. The first challenge is the traditional 'hub-and-spoke' design of telemedicine services where a tertiary hospital or 'hub' provides the technology and clinical expertise to support rural or 'outreach' sites. The scalability of this design is limited by the number of doctors in any given hub and the amount of time they can spend offering remote consultations. This is an absolute limitation beyond challenges such as legal and billing hurdles that restrict the use of remote consultations in Western countries. ${ }^{20}$

An alternative to a hub-and-spoke model is a distributed network of individual doctors and institutions to provide remote consultations. This model is currently being tested in Kenya $^{21}$ and involves a mix of local and global skills, including retired and diaspora doctors, medical missionaries and medical schools with global health track residency programmes, each with different, but powerful incentives to support healthcare in LMICs (figure 2). Consultations are requested through and mediated by locally registered medical professionals, typically nurses and clinical officers. Such mediated, 'provider-provider' remote consultations can be logistically simpler than direct 'patient-provider' remote consultations, expose healthcare professionals in LMICs to global knowledge and practices and potentially contribute to building local capacity. The distributed network limits the time required of any one provider and
Figure 1 Within-country disparities in human and hospital resources for health in Kenya, by county. The line represents the population of the 47 counties of Kenya (millions). Red bars represent the number of healthcare workers (nurses, clinical officers, doctors) per 1000 people in the public sector, which ranges from 1.56 in Isiolo county to 0.12 in Mandera county. ${ }^{15}$ Blue and green bars represent beds and cots (both public and private sectors) per 1000 people, respectively. Counties are arranged in descending order of beds-to-population ratio, from 3.48 beds per 1000 people in Isiolo to 0.39 in Mandera. Cots available range from 0.93 cots per 1000 people in Isiolo to 0.03 in Bungoma. $^{16}$

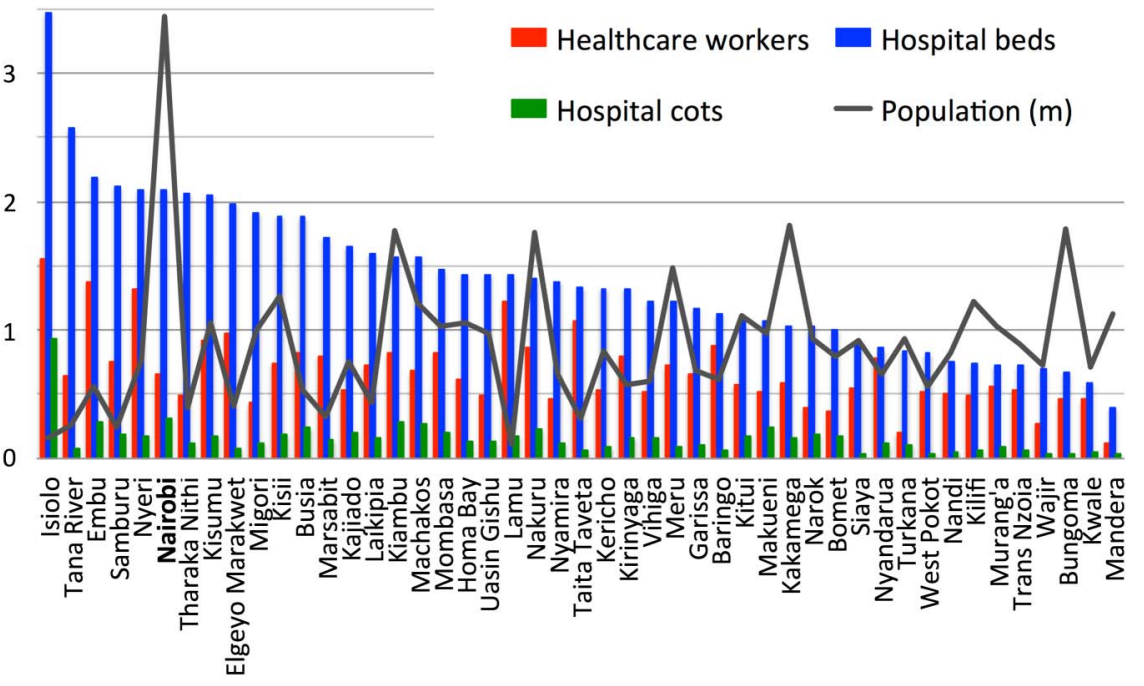




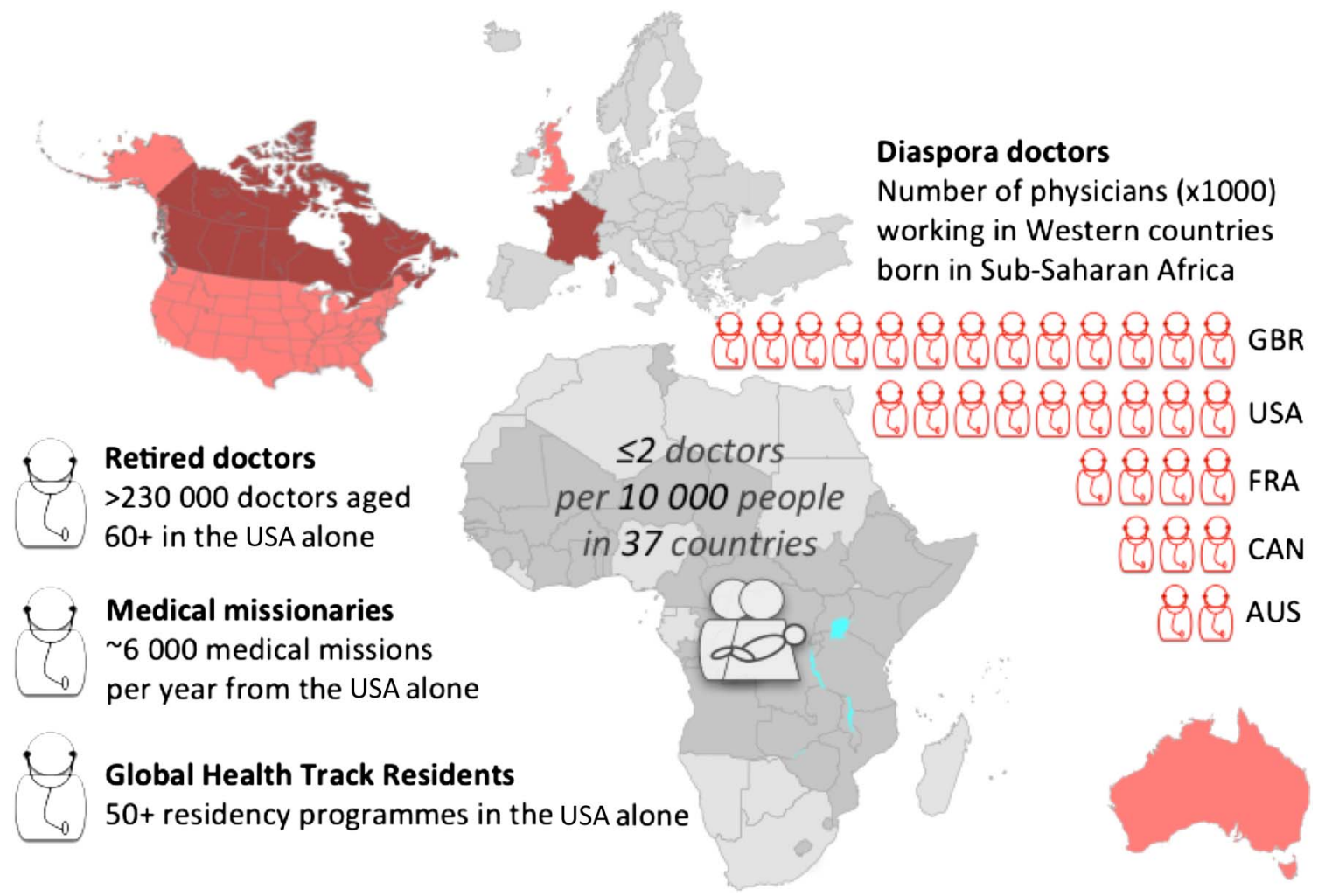

Figure 2 Illustration of the shortfall of doctors in low and middle income countries and global skills available to provide remote medical consultations. There are two or fewer doctors per 10000 people in 37 countries in Africa (including South Sudan). ${ }^{48}$ However, both local and global resources can be used to temporarily address this shortfall using telemedicine. These include diaspora doctors, ${ }^{49}$ retired doctors, ${ }^{50}$ those taking part in medical missions ${ }^{51}$ and residents enrolled in global health track programmes.

overcomes the constraint of the number of remote professionals available in any one centre. However, the service has limited control over turnaround times, making such 'network' telemedicine less suitable to emergency situations.

Other limitations of traditional telemedicine approaches include a reliance on 'real-time' video-based technology and the limited ability to digitise, store and share medical data as part of the consultation. The use of interactive video can be traced to the origins of telemedicine, ${ }^{19} 22$ and most instances of telemedicine expect multiple care providers and patient to engage simultaneously. This, however, is in contrast to much of consultative medicine, which is practised asynchronously, with clinicians leaving messages for each other, requesting tests and referrals. ${ }^{19}$ Real-time telemedicine implementations (eg, 'call-a-doctor' services or videoconferencing) often ignore the need for sharing medical records, limiting the meaningfulness of such consultations, or are paired with traditional EMRs-complex software that typically requires extensive training to use. With very limited use of EMRs in LMICs, the pairing of telemedicine with traditional EMRs is likely to limit its ability to scale in low-resource settings.

Possible solutions to these limitations include the asynchronous or 'store-and-forward' telemedicine model (rarely used beyond teleradiology or telepathology), ${ }^{23}{ }^{24}$ and EMRs based on the concept of a structured narrative. ${ }^{25}$ A narrative history is intuitive for healthcare workers to enter electronically with minimal training and provides important context and placeholders for structured digital data. A current implementation being tested by one of the authors is a tablet-based application combining efficient, offline entry of narrative information, creation of digital medical data from paper-based records (including tools to redact confidential information and link data to the narrative) and a communications platform to asynchronously interact with a distributed network of remote physicians. $^{21}$

\section{RUBBER STAMPS AND MOBILE PHONES-CHALLENGES AND SOLUTIONS TO ADHERENCE TO CLINICAL PRACTICE GUIDELINES}

There are other barriers beside shortages in HRH to delivering high-quality clinical care in LMICs. These are particularly evident in rapidly growing urban slums, where even within a setting considered relatively homogenous the quality of care can vary greatly. ${ }^{26}$ Improving adherence to clinical practice guidelines (CPGs) is one means of improving quality of care. However, there are challenges to developing guidelines relevant for use in low-resource settings and even greater ones to implementing and monitoring their use. ${ }^{27} 28$

To be effective, CPGs need to be (a) developed or adapted in an inclusive manner with direct input from the medical staff who will be implementing them, ${ }^{29}$ (b) included into existing workflows without requiring significant additional effort or $\operatorname{cost}^{30}$ and (c) paired with systems to measure, evaluate, feedback and reward evidence-based practice, thus motivating providers to deliver high-quality clinical care. ${ }^{31}$ While the impact of audits and feedback in LMIC healthcare systems can be high, ${ }^{32}$ one factor limiting the use of such methods is the high cost of performing audits, ${ }^{33}$ particularly in the absence of EMRs. The alternatives to clinical audits for collecting data on quality of clinical care in LMICs are also expensive, involving detailed interviews and observations, ${ }^{34}$ or standardised patients. ${ }^{35}$ 
A solution to some of these challenges of measuring and improving quality of clinical care is the use of checklists, as widely demonstrated in surgical settings. ${ }^{36}$ An extension of this concept being tested in Nairobi involves the use of rubber stamps to place checklists to support the diagnosis and management of various clinical conditions into paper case sheets for use during patient encounters. ${ }^{37}$ The Guideline Adherence in Slums Project (GASP) has prototyped rubber stamp CPG templates (RSTs) that are condition specific (eg, urinary tract infection (UTI), hypertension, etc) and incorporates important elements of relevant CPGs, diagnostic differentials and treatment options while accounting for the limitations of a particular clinic or clinical setting. The RSTs (figure 3) function both as a 'checklist', reminding providers of often-missed care (eg, screening for sexually transmitted infections in cases presenting as UTIs), and as a means to record important clinical information (eg, antibiotic used and justification for use). While RSTs are not intended to replace traditional paper-based case documentation, they are designed for easy digitisation using a mobile phone camera, rapidly capturing key clinical data in settings with limited resources. Early results from RST use show promising reductions in antibiotic prescription rates for common infections and other metrics of quality of clinical care, as well as enthusiastic support from providers using the tools. $^{37}$

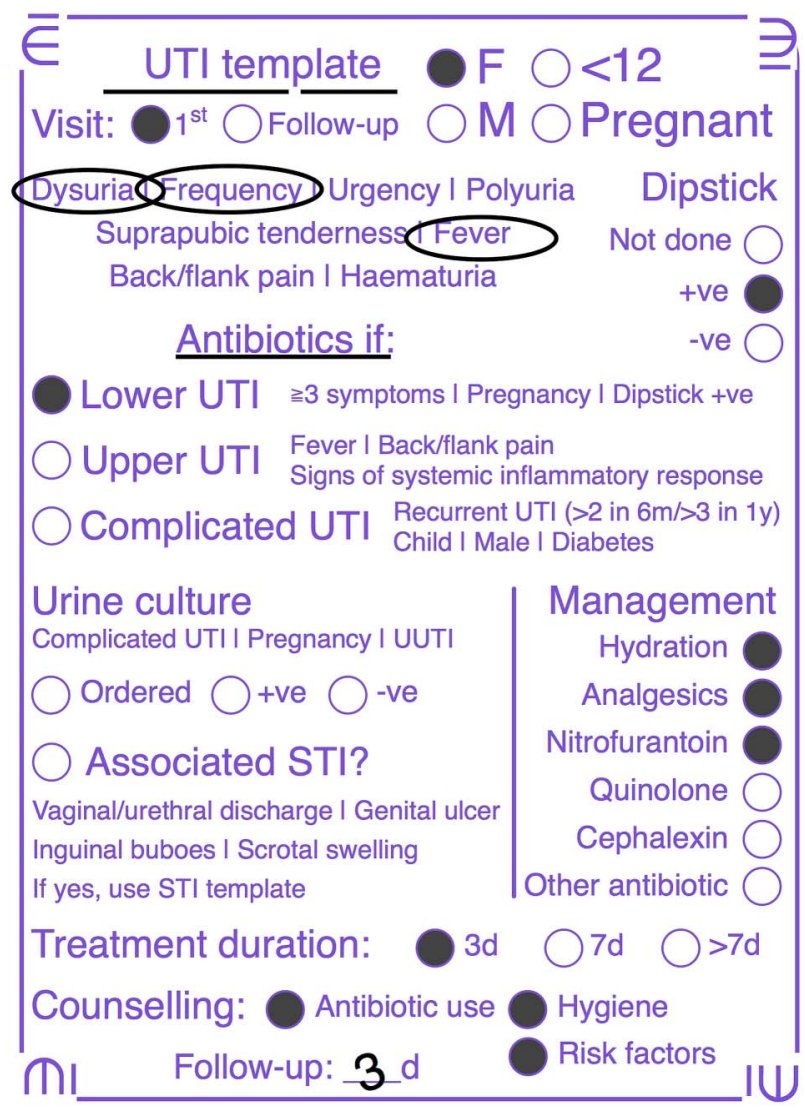

Figure 3 A to-scale illustration of a template for managing urinary tract infections (UTIs). The template is placed in the case sheet using a rubber stamp. Fiducial markings in the corners allow for accurate digitisation and image analysis using mobile phone cameras. The optical 'bubble' sheet format allows for rapid and efficient extraction and analysis of digital data from paper-based medical records.

\section{CONCLUSIONS}

The shortage of skilled HRH coupled with other challenges to document, manage and deliver high-quality healthcare in LMICs creates a fundamentally different setting for the design and deployment of e-Health solutions from those in highincome countries. At the individual level, almost every patient and provider in LMICs owns a mobile phone, so it is vital that mobile technologies play a part in improving healthcare. With ever-greater processing power in mobile devices and growing telephone and internet connectivity, the capacity for these devices to deliver change is unprecedented. However, many social challenges remain: for example, mobile phone ownership and usage in Kenya is associated with gender, level of education, literacy, urbanisation and the socioeconomic status of individuals. $^{38} 39$ Beyond these barriers to reaching consumers of healthcare (a major focus of e-Health technologies in highincome countries), the shortage of skilled HRH in LMICs also highlights the need for such tools to help empower healthcare providers in these settings. ${ }^{40} 41$

There are likely to be other differences in the ways e-Health interventions will be used in LMICs when compared with highincome countries. For example, with greater efforts being made in the standardisation of such technologies, it is likely that open source software will garner a larger proportion of the e-Health market. Hospital systems in LMICs are also likely to adopt 'mobile first' and patient-centred approaches to their technologies. As high-income countries adapt their existing PC-based systems to support mobile technology, it is possible that innovation in low-income settings could 'leap-frog' usability, connectivity and privacy issues that are causing serious problems in the high-income world due to legacy systems still in use. To succeed, however, e-Health solutions in LMICs also need to innovate on additional fronts to overcome the $\mathrm{HRH}$ and other challenges mentioned earlier.

The growing number of non-state actors in e-Health is also highlighting the challenges to effective regulation of healthcare technologies. Weak institutional capacity is recognised as a challenge to effective regulation of healthcare in LMICs, driving a rethink of the role of the state in regulation. ${ }^{42}$ In Kenya, the rapid advancement of the information \& communications technology (ICT) sector, coupled with the presence of a sizeable ICT human resource base, has contributed to increased implementation of e-Health projects in the country. As a result, in 2011, the Ministry of Health launched a National e-Health Strategy 2011-2017. ${ }^{43}$ The strategy describes how the Kenyan health system is faced with the challenge of rising cost and demand for quality healthcare services against a backdrop of shortages in skilled HRH. The e-Health strategy therefore highlights the gaps in healthcare services and aims to address these challenges by harnessing ICT. Five strategic areas have been identified: telemedicine, health information systems, m-Health, e-Learning and information for citizens. The strategy anticipates that ICT would promote and efficiently deliver healthcare services to Kenyans. The launch of the national e-Health strategy in Kenya has seen numerous public and private sector initiatives across the strategic areas. ${ }^{44} 45$

Driving change in low-resource settings also requires appropriate 'change agents'. For-profit enterprises and related investment vehicles have traditionally been the agents that design and deliver sustainable, demand-driven solutions. However, improving healthcare for underserved populations in LMICs may not generate the large margins required to attract traditional enterprises. But the inherent value in private sector enterprise 
methods has resulted in a proliferation of 'social enterprises'businesses with social objectives. ${ }^{46}$ Social enterprises could prove to be the change agents for the innovation needed in e-Health, merging entrepreneurship with efficient financing and operations needed for delivering impact that is both sustainable and scalable.

E-Health solutions in healthcare, especially in LMICs, are still in their infancy. It is a time for policymakers, funders and regulators in LMICs to actively encourage innovation and entrepreneurship, the main vehicles driving change. Regulation will naturally follow in the wake of innovations, encouraging changes that benefit society while protecting its citizens. ${ }^{47}$

Twitter Follow Pratap Kumar at @healthEnet

Acknowledgements The authors are grateful to Meghan Bruce Kumar for comments on the manuscript, Dr Bernadette Kleczka for work on template development, to Emily Bowler for research assistance and to colleagues at Health-E-Net, Strathmore Business School and the KEMRI-Wellcome Trust Research Programme for their support.

Contributors PK coordinated developing the initial draft of this publication and prepared the final draft after input from coauthors. All authors approved the final version.

Funding PK is funded by a Stars in Global Health award (\$5 0420-01) from Grand Challenges Canada. PK and CP are funded by Health Systems Research Initiative grants (MR/N005015/1; MR/N005600/1) jointly supported by the Department for International Development (DFID), the Economic and Social Research Council (ESRC), the Medical Research Council (MRC) and the Wellcome Trust (WT). DK is funded by a grant from the Canadian International Development Research Centre (IDR-AHI01).

Competing interests PK is the CEO of Health-E-Net, a healthcare social enterprise in Kenya.

Provenance and peer review Commissioned; externally peer reviewed.

\section{REFERENCES}

1 Weiner JP, Yeh S, Blumenthal D. The impact of health information technology and e-health on the future demand for physician services. Health Aff (Millwood) 2013;32:1998-2004.

2 Obasola OI, Mabawonku I, Lagunju I. A Review of e-Health Interventions for Maternal and Child Health in Sub-Sahara Africa. Matern Child Health J 2015:19:1813-24.

3 Déglise C, Suggs LS, Odermatt P. SMS for disease control in developing countries: a systematic review of mobile health applications. J Telemed Telecare 2012;18:273-81.

4 Lewis T, Synowiec C, Lagomarsino G, et al. E-health in low- and middle-income countries: findings from the Center for Health Market Innovations. Bull World Health Organ 2012;90:332-40.

5 Communications Authority of Kenya. First quarter sector statistics report for the financial year 2015/2016 (July-September 2015). http://www.ca.go.ke/images/ downloads/STATISTICS/Sector\%20\%20Statistics\%20Report\%20Q1\%202015-16.pdf (accessed 3 Apr 2016).

6 van Gemert-Pijnen JE, Nijland N, van Limburg M, et al. A Holistic framework to improve the uptake and impact of eHealth Technologies. J Med Internet Res 2011;13:e111.

7 Alpay LL, Henkemans OB, Otten W, et al. E-health applications and services for patient empowerment: directions for best practices in The Netherlands. Telemed J E Health 2010;16:787-91.

8 Tomlinson M, Rotheram-Borus MJ, Swartz L, et al. Scaling up mHealth: where is the evidence? PLoS Med 2013;10:e1001382.

9 Blumenthal D, Tavenner M. The "meaningful use" regulation for electronic health records. N Engl J Med 2010;363:501-4.

10 Greenhalgh T, Keen J. England's national programme for IT. BMJ 2013;346:f4130.

11 Kuperman GJ. Health-information exchange: why are we doing it, and what are we doing? J Am Med Inform Assoc 2011;18:678-82.

12 Tierney WM, Achieng $M$, Baker $E$, et al. Experience implementing electronic health records in three East African countries. Stud Health Technol Inform 2010;160:371-5

13 Fritz F, Tilahun B, Dugas M. Success criteria for electronic medical record implementations in low-resource settings: a systematic review. J Am Med Inform Assoc 2015;22:479-88.

14 Campbell J, Dussault G, Buchan J, et al. A universal truth: no health without a workforce. Forum Report, Third Global Forum on Human Resources for Health, Recife, Brazil. Geneva: Global Health Workforce Alliance and World Health Organization, 2013
15 Ministry of Health, Government of Kenya. Integrated payroll and personnel database. 2012

16 Ministry of Health, Government of Kenya. Master Facility List. http://ehealth.or.ke/ facilities/download-latest.aspx (accessed 28 Jul 2015).

17 Temu F, Leonhardt M, Carter J, et al. Integration of non-communicable diseases in health care: tackling the double burden of disease in African settings. Pan Afr Med J 2014;18:202.

18 Ministry of Health, Government of Kenya. 2013 Kenya household health expenditure and utilisation survey. Nairobi: Government of Kenya, 2014.

19 Field MJ, ed. Telemedicine: a guide to assessing telecommunications in health care. Washington DC: National Academy Press, 1996.

20 American Telemedicine Association. State Telemedicine Gaps Analysis. http://www. americantelemed.org/policy/state-policy-resource-center\#.VewSsZ2qqko (accessed 31 Aug 2015).

21 http://www.grandchallenges.ca/grantee-stars/0420-01/

22 Freiburger G, Holcomb M, Piper D. The STARPAHC collection: part of an archive of the history of telemedicine. J Telemed Telecare 2007;13:221-3.

23 Allely EB. Synchronous and asynchronous telemedicine. J Med Syst 1995:19:207-12

24 von Wangenheim A, de Souza Nobre LF, Tognoli H, et al. User satisfaction with asynchronous telemedicine: a study of users of Santa Catarina's system of telemedicine and telehealth. Telemed J E Health 2012;18:339-46.

25 Johnson SB, Bakken S, Dine D, et al. An electronic health record based on structured narrative. J Am Med Inform Assoc 2008;15:54-64.

26 More NS, Bapat U, Das S, et al. Inequalities in maternity care and newborn outcomes: one-year surveillance of births in vulnerable slum communities in Mumbai. Int J Equity Health 2009;8:21.

27 Rowe SY, Kelly JM, Olewe MA, et al. Effect of multiple interventions on community health workers' adherence to clinical guidelines in Siaya district, Kenya. Trans $R$ Soc Trop Med Hyg 2007;101:188-202.

28 Agweyu A, Opiyo N, English M. Experience developing national evidence-based clinical guidelines for childhood pneumonia in a low-income setting - making the GRADE? BMC Pediatr 2012;12:1.

29 Higuchi M, Okumura J, Aoyama A, et al. Application of standard treatment guidelines in rural community health centres, Timor-Leste. Health Policy Plan 2012;27:396-404.

30 Kawamoto K, Houlihan CA, Balas EA, et al. Improving clinical practice using clinical decision support systems: a systematic review of trials to identify features critical to success. BMJ 2005:330:765.

31 English M, Nzinga J, Mbindyo $P$, et al. Explaining the effects of a multifaceted intervention to improve inpatient care in rural Kenyan hospitals -interpretation based on retrospective examination of data from participant observation, quantitative and qualitative studies. Implement Sci 2011;6:124.

32 Jamtvedt G, Young JM, Kristoffersen DT, et al. Audit and feedback: effects on professional practice and health care outcomes. Cochrane Database Syst Rev 2006; (2):CD000259.

33 Lock P, McElroy B, Mackenzie M. The hidden cost of clinical audit: a questionnaire study of NHS staff. Health Policy 2000;51:181-90.

34 Rannan-Eliya RP, Wijemanne N, Liyanage IK, et al. The quality of outpatient primary care in public and private sectors in Sri Lanka-how well do patient perceptions match reality and what are the implications? Health Policy Plan 2015;30(Suppl 1): i59-74.

35 Das J, Holla A, Das V, et al. In urban and rural India, a standardized patient study showed low levels of provider training and huge quality gaps. Health Affairs 2012:31:2774-84.

36 Haynes AB, Weiser TG, Berry WR, et al. A surgical safety checklist to reduce morbidity and mortality in a global population. $N$ Engl J Med 2009;360:491-9

37 Kumar P, Kleczka B, Ngunjiri D, et al. The Guidelines Adherence in Slums Project (GASP). 1st Annual Regional eHealth Conference, Ministry of Health, Kenya, 2015

38 Wesolowski A, Eagle N, Noor AM, et al. Heterogeneous mobile phone ownership and usage patterns in Kenya. PLOS ONE 2012;7:e35319.

39 Zurovac D, Otieno G, Kigen S, et al. Ownership and use of mobile phones among health workers, caregivers of sick children and adult patients in Kenya: cross-sectional national survey. Global Health 2013;9:20.

40 Free C, Phillips G, Galli L, et al. The effectiveness of mobile-health technology-based health behaviour change or disease management interventions for health care consumers: a systematic review. PLoS Med 2013;10: e1001362.

41 Free C, Phillips G, Watson L, et al. The effectiveness of mobile-health technologies to improve health care service delivery processes: a systematic review and meta-analysis. PLoS Med 2013;10:e1001363.

42 Akhtar A. Health care regulation in low-and middle-income countries: a review of the literature. Melbourne: The Nossal Institute for Global Health. Health policy and health finance knowledge hub working paper series, 2011:14.

43 Republic of Kenya, Kenya National e-Health Strategy 2011-2017, Ministry of Medical Services and Ministry of Public Health and Sanitation, Editors. Nairobi, 2011. 
44 Talbot D. Kenya's mobile prescription: local programmers and homegrown business models are helping to realize the vast promise of using phones to improve health care and save lives. Technol Rev (Camb, Mass) 2012;115:52.

45 Juma K, Nahason M, Apollo W, et al. Current status of E-Health in Kenya and Emerging Global Research Trends. Int J Inform Commun Technol Res 2012;2:50.

46 Roy MJ, Donaldson C, Baker R, et al. The potential of social enterprise to enhance health and well-being: a model and systematic review. Soc Sci Med 2014;123:182-93.

47 Jamard A. Innovation in regulation turmoil. Wired 2014. http://www.wired.com/ insights/2014/06/innovation-regulation-turmoil/ (accessed 31 Aug 2015).
48 Naicker S, Plange-Rhule J, Tutt RC, et al. Shortage of healthcare workers in developing countries-Africa. Ethn Dis 2009;19(Suppl 1): S1-60-64.

49 Clemens MA, Pettersson G. New data on African health professionals abroad. Hum Resour Health 2008;6:1.

50 Young A, Chaudhry HJ, Pei $X$, et al. A census of actively licensed physicians in the United States, 2010. J Med Regul 2011;101:8-23.

51 Maki J, Qualls M, White B, et al. Health impact assessment and short-term medical missions: a methods study to evaluate quality of care. BMC Health Serv Res 2008;8:121. 